\title{
Scintillation Properties of Sn-doped Yttrium Aluminum Garnet (YAG)
}

\author{
Takayuki Yanagida, ${ }^{1 *}$ Hirokazu Masai, ${ }^{2}$ Masanori Koshimizu, ${ }^{3}$ and Noriaki Kawaguchi ${ }^{1}$ \\ ${ }^{1}$ Nara Institute of Science and Technology, \\ 8916-5 Takayama, Ikoma, Nara 630-0196, Japan \\ ${ }^{2}$ National Institute of Advanced Industrial Science and Technology, \\ 1-8-31 Midorigaoka, Ikeda, Osaka 563-8577, Japan \\ ${ }^{3}$ Department of Applied Chemistry, Graduate School of Engineering, Tohoku University, \\ 6-6-07 Aoba, Aramaki, Aoba-ku, Sendai 980-8579, Japan
}

(Received Novcember 26, 2018; accepted March 25, 2019)

Keywords: scintillator, YAG, floating zone, scintillation detector, photoluminescence

By using the floating zone method, we synthesized Sn-doped yttrium aluminum garnet (YAG). The nominal concentration of Sn was $10 \%$ with respect to $\mathrm{Y}$, and the sample rod was cut along the growth direction. By the segregation phenomenon, we finally obtained Sn-0.6-, 1.0-, 1.2-, 1.4-, and 3.0\%-doped YAG specimens with a diameter of $3 \mathrm{~mm}$ and a thickness of $1 \mathrm{~mm}$. When we irradiated them with X-rays, a broad emission peaking at $420 \mathrm{~nm}$ was observed, and a similar spectral shape was observed in the photoluminescence (PL) emission spectrum. The decay times in PL and scintillation were 5 to $10 \mu \mathrm{s}$. Taking into account these data and possible emission origins in this chemical composition, we concluded the emission origin to be $\mathrm{Sn}^{2+}$.

\section{Introduction}

Scintillators are one of the phosphor materials that have the function of converting the invisible ionizing radiation to visible photons, ${ }^{(1)}$ and the range of the application of such scintillation detectors has been wide, including medical, ${ }^{(2)}$ well-logging, ${ }^{(3)}$ environmental monitoring, ${ }^{(4)}$ and astrophysics, ${ }^{(5)}$ and particle physics. ${ }^{(6)}$ In the recent trend, scintillators generally consist of the host material and emission center. The former is responsible for absorbing ionizing radiation, and the preferable chemical composition of the host depends on the target ionizing radiation species. In the latter, the emission centers emit scintillation photons, the number of which is proportional to the absorbed energy. Mostly, rare-earth ions are selected as the emission center in scintillators ${ }^{(7)}$ since some of the rare-earth ions can show a bright emission due to $5 \mathrm{~d}-4 \mathrm{f}$ transitions, and rare-earth-ion-doped materials are widely used in phosphor applications ${ }^{(8-11)}$ as well as scintillation detectors.

In addition to rare-earth ions, other emission centers have been tested. The most common approach is to use transition metal ions. In particular, investigations of Mn-doped materials can be seen frequently. ${ }^{(12-14)}$ The other solution is to use $\mathrm{ns}^{2}$ ions as emission centers since

*Corresponding author: e-mail: t-yanagida@ms.naist.jp

https://doi.org/10.18494/SAM.2019.2181 
these ions have been known to show bright emissions. ${ }^{(15-17)} \mathrm{Sn}^{2+}$ has attracted much attention because of a high photoluminescence (PL) quantum yield (QY) that reaches up to $\sim 100 \%$. ${ }^{(18-20)}$ However, most studies on Sn-doped phosphors have been done in glass materials, and there still remains room to study for crystalline materials.

In this work, we investigate Sn-doped yttrium aluminum garnet $\left(\mathrm{Y}_{3} \mathrm{Al}_{5} \mathrm{O}_{12}\right.$; $\left.\mathrm{YAG}\right)$ because YAG is a very common host in phosphor materials. ${ }^{(21-23)}$ To our knowledge, there have been no studies on Sn-doped YAG. Optical properties, including PL emission and excitation spectra, PL QY, and decay time, and scintillation properties, such as X-ray-induced radioluminescence (RL) and decay, were investigated.

\section{Materials and Methods}

The samples were synthesized by the floating zone (FZ) method. First, raw materials of $\mathrm{Y}_{2} \mathrm{O}_{3}(4 \mathrm{~N}), \mathrm{Al}_{2} \mathrm{O}_{3}(4 \mathrm{~N})$, and $\mathrm{SnO}_{2}(4 \mathrm{~N})$ were mixed homogeneously to achieve Sn-10\%doped $\mathrm{Y}_{3} \mathrm{Al}_{5} \mathrm{O}_{12}$. Here, $\mathrm{Sn}$ was added with respect to $\mathrm{Y}$. The mixed powder was formed into a cylindrical shape by hydrostatic pressure, and the cylindrical rod was sintered at $1500{ }^{\circ} \mathrm{C}$ for 12 $\mathrm{h}$. The sintered rod was loaded into the FZ furnace, and we grew the crystal by the FZ method with pull-down and rotation rates of $5 \mathrm{~mm} / \mathrm{h}$ and $20 \mathrm{rpm}$, respectively.

Generally, all the melt growth techniques cause the segregation of the dopant ions, and there must be a dopant concentration gradient along the growth direction. If we cut the sample rod perpendicularly to the growth direction, we can obtain several samples with different dopant concentrations. This concept was previously tested in Ce-doped $\mathrm{YAlO}_{3}$ (YAP), and we confirmed the validity. ${ }^{(24)}$ Figure 1(a) shows the grown sample, and Fig. 1(b) shows examples of cut samples. The grown sample looked semitransparent, and the semitransparency was also observed in cut sample pieces. The size of each cut sample piece was $3 \mathrm{~mm} \phi \times 1 \mathrm{mmt}$. The Sn concentration was evaluated by X-ray fluorescence (XRF) using MESA-500W (HORIBA). We could obtain samples with Sn concentrations of $0.6,1.0,1.2,1.4$, and $3.0 \%$ at one time. The surfaces of the samples were polished to have the same thickness.

The cut samples were characterized as follows. As optical properties, PL excitation and the emission contour graph were observed using the Quantaurus-QY spectrometer (Hamamatsu). The PL decay time was evaluated using Quantaurus- $\tau$ (Hamamatsu) with two different cases of wavelength excitation and monitoring. One was monitoring at $400 \mathrm{~nm}$ under $280 \mathrm{~nm}$ excitation, and the other was monitoring at $600 \mathrm{~nm}$ under $340 \mathrm{~nm}$ excitation. The decay time was deduced by the approximation of a single exponential component or the sum of two exponential components.

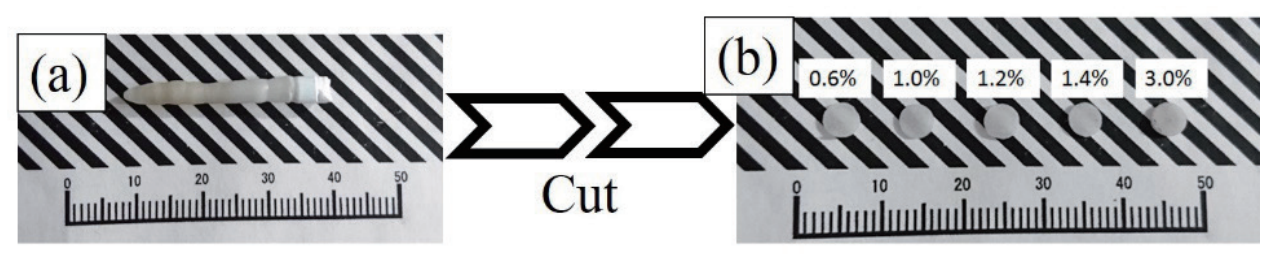

Fig. 1. (a) As-grown sample and (b) cut sample pieces. 
As scintillation properties, X-ray-induced scintillation spectra were observed in our original setup. ${ }^{(25)}$ The X-ray generator (XRB80N100/CB, Spellman) used was equipped with an ordinary X-ray tube having a tungsten anode target and a beryllium window. The applied bias voltage and tube current were $80 \mathrm{kV}$ and $1.2 \mathrm{~mA}$, respectively. During the X-ray irradiation, the scintillation photons from the sample were collected and guided to the spectrometer (Andor DU420-BU2 CCD with a Shamrock SR163 monochromator) through a $2 \mathrm{~m}$ optical fiber to measure the spectrum. We have measured the scintillation decay time profiles using a pulsed X-raysource-equipped afterglow characterization system ${ }^{(26)}$ that allowed decay curve measurements by the time-correlated-single-photon-counting (TCSPC) technique. The wavelength sensitivity of the photomultiplier tube (PMT) used in this measurement was from 160 to $650 \mathrm{~nm}$, and the analysis methodology was the same as that in PL decay.

\section{Results and Discussion}

Figure 2 shows the PL excitation versus emission contour graph of the Sn 1.0\% sample as an example. We can observe broad emission bands from 320 to $550 \mathrm{~nm}$ under $280-300 \mathrm{~nm}$ excitation (emission band 1) and from 400 to $700 \mathrm{~nm}$ under 260-350 nm excitation (emission band 2). All the samples showed similar spectral shapes, although the intensity (contrast) differed. As the emission intensity, we evaluated the PL QY and found the value to be $\sim 1 \%$ in all the samples. Compared with conventional Ce-doped YAG, which has a QY of $\sim 90 \%$, the emission intensity was low. The interesting feature was the broad emission band, which contained all the visible wavelengths from 400 to $650 \mathrm{~nm}$. This spectral feature resembled that of Sn-doped glass phosphors. ${ }^{(18-20)}$

To consider the emission origin further, we have measured the PL decay time under two different excitation/monitoring conditions, as described above. Figure 3(a) shows decay curves monitored at $400 \mathrm{~nm}$ under $280 \mathrm{~nm}$ excitation. All the samples exhibited similar decay curves consisting of fast (1-2 $\mu \mathrm{s})$ and slow $(\sim 5 \mu \mathrm{s})$ components. When the $\mathrm{Sn}$ concentration increased, the decay time became shorter. The tendency observed here was also typical in Sn-doped glass phosphors. ${ }^{(18-20)}$ On the other hand, Fig. 3(b) exhibits decay curves monitored at $600 \mathrm{~nm}$ under $340 \mathrm{~nm}$ excitation. No systematic tendency related to Sn concentrations was observed, and all the samples showed the same decay time of $\sim 6.5 \mu$ s. Such a component was not observed in Sn-

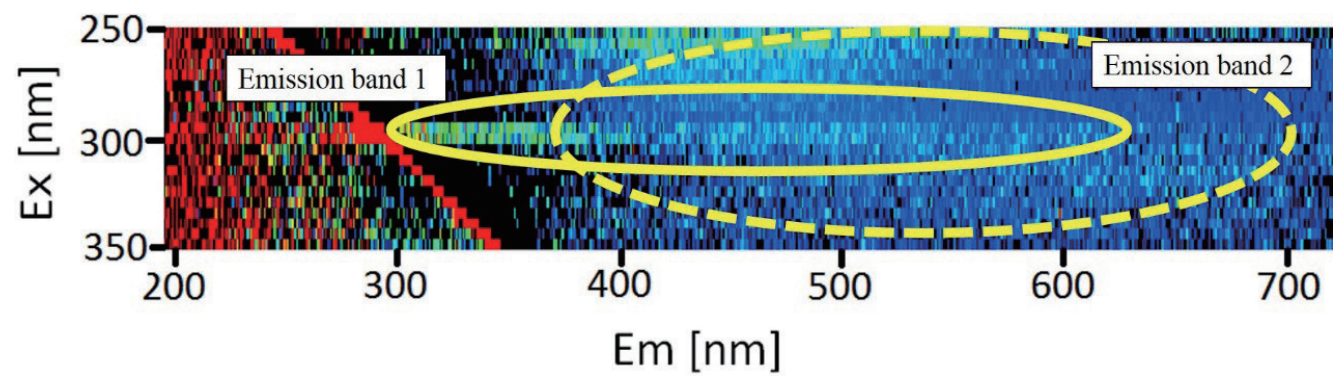

Fig. 2. (Color online) PL excitation (vertical axis) versus emission (horizontal axis) contour graph of Sn-1.0\%doped sample. 
(a) PL $\lambda \mathrm{ex}=280 \mathrm{~nm}, \lambda \mathrm{em}=400 \mathrm{~nm}$

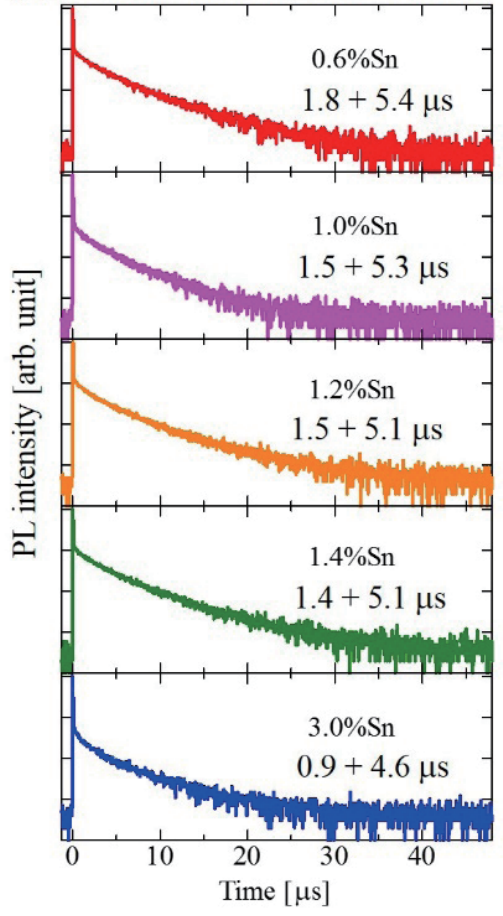

(b) PL $\lambda \mathrm{ex}=340 \mathrm{~nm}, \lambda \mathrm{em}=600 \mathrm{~nm}$

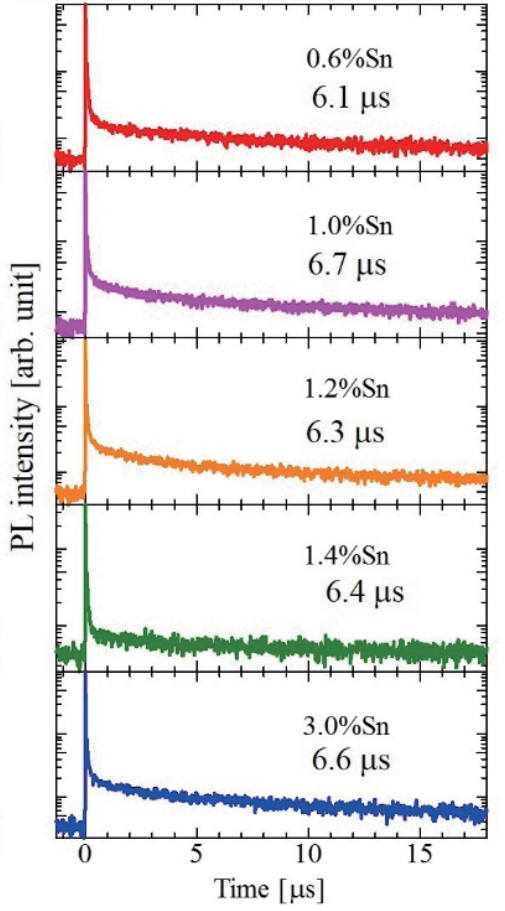

Fig. 3. (Color online) PL decay curves monitored at (a) $400 \mathrm{~nm}$ under $280 \mathrm{~nm}$ excitation and (b) at $600 \mathrm{~nm}$ under $340 \mathrm{~nm}$ for all samples.

doped glass phosphors. ${ }^{(18-20)}$ In both measurements, the very fast (spikelike) component was due to the instrumental response in the monitoring time ranges.

Referring to these experimental data, we considered the possible emission origin. Possible emission origins are luminescence from (i) $\mathrm{Sn}^{2+}$, (ii) unexpected rare-earth (RE) contamination, (iii) unexpected transitional metal (TM) contamination, and (iv) the YAG host. At least in XRF measurement, we could not confirm unexpected contamination. Thus, if such unexpected contamination occurred, the concentration would be less than $0.1 \%$. The possibility of (ii) unexpected RE contamination can be rejected easier. Such a broad emission band is possible with $\mathrm{Ce}^{3+},{ }^{(27)} \mathrm{Pr}^{3+}{ }^{2}{ }^{(28)}$ and $\mathrm{Eu}^{2+}{ }^{29)}$ but the observed decay times and emission wavelengths largely differed. Although the scenario of (iii) the unexpected contamination of TM may be possible in view of the spectral shape because the $d-d$ transition of TM can cause a broad emission, we can reject this possibility on the basis of the decay time. Generally, the decay time of the d-d transition of TM ions is in the millisecond range. The remaining possibility of (iv) the YAG host as the emission origin can also be rejected since the self-trapped exciton luminescence of YAG is around $300 \mathrm{~nm} .{ }^{(30)}$ Therefore, we can assign $320-550 \mathrm{~nm}$ emission to $\mathrm{Sn}^{2+}$ emission. On the other hand, the origin of $400-700 \mathrm{~nm}$ emission is not clear. Previously, 700-800 nm emission was observed in $\mathrm{YAG}^{(31)}$ but our results did not coincide with them. The previous report showed the excitation and emission wavelengths of 190 and $700 \mathrm{~nm}$, respectively. The possible interpretation of this emission band from the spectral information 
would be ascribed to $\mathrm{Sn}^{4+}$ or some kind of defect generated during the crystal growth by the charge imbalance of $\mathrm{Sn}^{2+} / \mathrm{Sn}^{4+}$ with $\mathrm{Y}^{3+}$. By taking into account that these samples did not show any clear Sn concentration dependence of decay time, the defect origin would be possible.

Figure 4 shows X-ray-induced scintillation spectra of all the samples. All the samples showed the same spectral shape with a peak at $420 \mathrm{~nm}$, although the emission intensities were different. In all the samples, a sharp line at $310 \mathrm{~nm}$ was detected, and it was ascribed to unexpected $\mathrm{Gd}$ contamination since the $4 \mathrm{f}-4 \mathrm{f}$ transition of $\mathrm{Gd}^{3+}$ has exhibited this characteristic line at $310 \mathrm{~nm}$ in many materials. ${ }^{(32-34)}$ A very broad emission band can be understood to mean that the excitation by ionizing radiation opens all kinds of excitation/emission channels, and we can interpret the observed spectra as a superposition of the two broad emission bands shown in PL spectra. Among the present samples tested here, the Sn-0.6\%-doped sample exhibited the highest scintillation intensity. From the viewpoint of a scintillation detector, the observed emission wavelength is acceptable since the wavelength agrees well with the sensitive wavelength range of conventional PMTs and Si photodiodes.

Figure 5 shows X-ray-induced scintillation decay time profiles of all the samples. All the decay curves can be reproduced by the approximation of the sum of three exponential components. The observed decay curves are characterized by the fast, middle, and slow components. The origin of the fast component is the instrumental response in this time range, and the middle component is attributed to the host luminescence of YAG since a similar (sub- $\mu \mathrm{s}$ ) component is often observed in the scintillation of doped and undoped YAG. ${ }^{(30)}$ The origin of

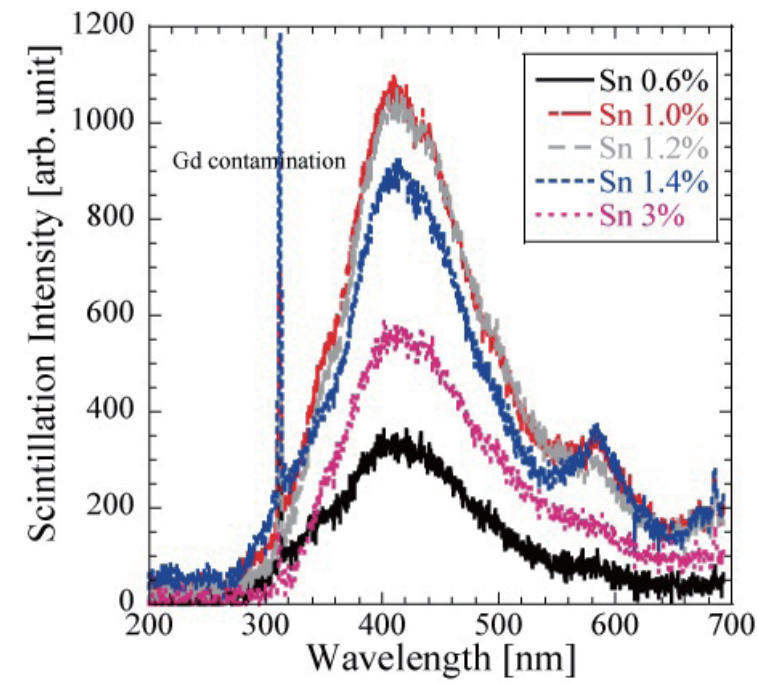

Fig. 4. (Color online) X-ray-induced scintillation spectra of all the samples.

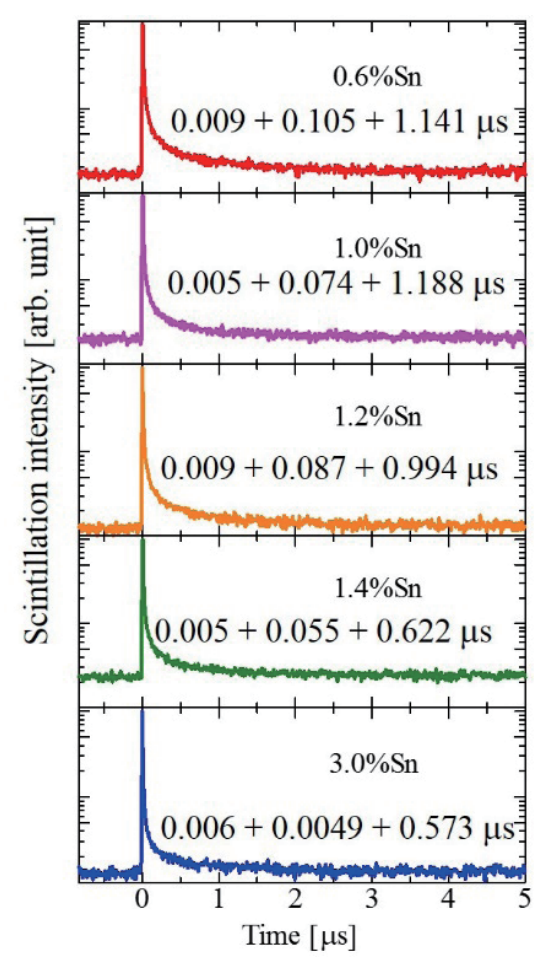

Fig. 5. (Color online) X-ray-induced scintillation decay time profiles of all the samples. 
the slow component is the $\mathrm{ns}^{2}$-nsnp transition of $\mathrm{Sn}^{2+}$ because the observed decay time is close to those in PL (1-2 $\mu \mathrm{s})$ and the decay time becomes shorter with increasing Sn concentration. From the viewpoint of a scintillation detector, the observed decay time is acceptable.

\section{Conclusions}

We synthesized Sn-0.6-, 1.0-, 1.2-, 1.4-, and 3.0\%-doped YAG samples by the FZ method. In PL, we confirmed the emission due to the $\mathrm{ns}^{2}-\mathrm{nsnp}$ of $\mathrm{Sn}^{2+}$ at $320-550 \mathrm{~nm}$ and a possible defect emission at around 400-700 nm. In X-ray-induced scintillation, the emission wavelength appeared at around $420 \mathrm{~nm}$ with a typical decay time of $\sim 1 \mu \mathrm{s}$. The present work shows the merits of Sn doping on common host materials of phosphor applications.

\section{Acknowledgments}

This work was supported by Grants-in-Aid for Scientific Research (A) (17H01375) and (B) (18H03468) from the Ministry of Education, Culture, Sports, Science and Technology of the Japanese government (MEXT). The Cooperative Research Project of the Research Institute of Electronics, Shizuoka University, Terumo Foundation for Life Sciences and Arts, Izumi Science and Technology Foundation, The Kazuchika Okura Memorial Foundation, and The Iwatani Naoji Foundation are also acknowledged.

\section{References}

1 T. Yanagida: Proc. Jpn. Academy, B 94 (2018) 75.

2 Y. Hirata, K. Watanabe, S. Yoshihashi, A. Uritani, Y. Koba, N. Matsufuji, T. Yanagida, T. Toshito, and K. Fukuda: Sens. Mater. 29 (2017) 1455.

3 T. Yanagida, Y. Fujimoto, S. Kurosawa, K. Kamada, H. Takahashi, Y. Fukazawa, M. Nikl, and V. Chani: Jpn. J. Appl. Phys. 52 (2013) 076401.

4 K. Watanabe, T. Yanagida, K. Fukuda, A. Koike, T. Aoki, and A. Uritani: Sens. Mater. 27 (2015) 269.

5 H. Takahashi, T. Yanagida, D. Kasama, T. Ito, M. Kokubun, K. Makishima, T. Yanagitani, H. Yagi, T. Shigeta, and T. Ito: IEEE Trans. Nucl. Sci. 53 (2006) 2404.

6 T. Ito, T.Yanagida, M. Sato, M. Kokubun, T. Takashima, S. Hirakuri, R. Miyawaki, H. Takahashi, K. Makishima, T. Tanaka, K. Nakazawa, T. Takahashi, and T. Honda: Nucl. Instrum. Methods Phys. Res., Sect. A 579 (2007) 239.

7 T. Yanagida: Opt. Mater. 35 (2013) 1987.

8 L. Zhang, Y. Xia, X. Shen, R. Yang, and W. Wei: Opt. Mater. 75 (2018) 1.

9 B. V. Padlyak, R. Lisiecki, T. B. Padlyak, and V. T. Adamiv: J. Lumin. 198 (2018) 183.

10 W. C. Lee, H. K. Yang, M. Kwak, and B. K. Moon: Optik 172 (2018) 1205.

11 K. A. Gedekar, S. P. Wankhede, S. V. Moharil, and R. M. Belekar: J. Mater. Sci.: Mater. Electron. 29 (2018) 4466.

12 X. Y. Sun, Z. He, and X. Gu: J. Mater. Sci.: Mater. Electron. 29 (2018) 6961.

13 R. Cao, W. Wang, J. Zhang, Y. Ye, T. Chen, S. Guo, F. Xiao, and Z. Luo: Opt. Mater. 79 (2018) 223.

14 P. Yu, and L. Tian: Opt. Mater. 85 (2018) 444.

15 S. Honda, A. Nohtomi, K. Machidori, and G. Wakabayashi: Nucl. Instrum. Methods Phys. Res., Sect. A 871 (2017) 141.

16 Y. Usui, T. Oya, G. Okada, N. Kawaguchi, and T. Yanagida: Mater. Res. Bull. 90 (2017) 266.

17 M. Jeong, B. Van, B. T. Wells, L. J. D’Aries, and M. D. Hammig: Nucl. Instrum. Methods Phys. Res., Sect. A 893 (2018) 75.

18 H. Masai, Y. Yamada, Y. Suzuki, K. Teramura, Y. Kanemitsu, and T. Yoko: Sci. Rep. 3 (2013) 3541. 
19 H. Masai, T. Tanimoto, S. Okumura, K. Teramura, S. Matsumoto, T. Yanagida, Y. Tokuda, and T. Yoko: J. Mater. Chem. C 2 (2014) 2137.

20 H. Masai, H. Miyata, Y. Yamada, S. Okumura, T. Yanagida, and Y. Kanemitsu: Sci. Rep. 5 (2015) 11224.

21 F. Mohammadi, O. Mirzaee, and M. Tajally: Opt. Mater. 85 (2018) 174.

22 G. Okada, N. Kawaguchi, and T. Yanagida: Sens. Mater. 29 (2017) 1407.

23 M. Łukaszewicz, and W. Stręk: J. Lumin. 199 (2018) 251.

24 A. Fukabori, T. Yanagida, F. Moretti, Y. Yokota, R. Shimura, S. Maeo, J. Pejchal, K. Kamada, and A. Yoshikawa: Radiat. Meas. 45 (2010) 453.

25 T. Yanagida, K. Kamada, Y. Fujimoto, H. Yagi, and T. Yanagitani: Opt. Mater. 35 (2013) 2480.

26 T. Yanagida, Y. Fujimoto, T. Ito, K. Uchiyama, and K. Mori: Appl. Phys. Exp. 7 (2014) 062401.

27 X. He, X. Liu, R. Li, B. Yang, K. Yu, M. Zeng, and R. Yu: Sci. Rep. 6 (2016) 22238.

28 R. Piramidowicz, K. Ławniczuk, M. Nakielska, J. Sarnecki, and M. Malinowski: J. Lumin. 128 (2008) 708.

29 Q. Q. Zhu, W. W. Hu, L. C. Ju, L. Y. Hao, X. Xu, and S. Agathopoulos: J. Am. Ceram. Soc. 96 (2013) 701.

30 Y. Fujimoto, T. Yanagida, H. Yagi, T. Yanagitani, and V. Chani: Opt. Mater. 36 (2014) 1926.

31 C. R. Varney, S. M. Reda, D. T. Mackay, M. C. Rowe, and F. A. Selim: AIP Adv. 1 (2011) 042170.

32 F. Nakamura, T. Kato, G. Okada, N. Kawano, N. Kawaguchi, K. Fukuda, and T. Yanagida: Mater. Res. Bull. 98 (2017) 83.

33 B. Fan, S. Qi, W. Zhao, S. Li, and S. An: J. Lumin. 196 (2018) 520.

34 B. V. Padlyak, A. Drzewiecki, T. B. Padlyak, V. T. Adamiv, and I. M. Teslyuk: Opt. Mater. 79 (2018) 302. 